\title{
Comparative Study of Imaging Characteristics of I-125 Imaging Using the Siemens Inveon Scanner and Siemens Symbia TruePoint
}

\author{
Young Jun Kim1, Ilhan Lim ${ }^{1,2 *}$, A Ram Yu1, Byung Il Kim¹,2, Chang Woon Choi1,2, \\ Sang Moo Lim ${ }^{1,2}$, Jin Su Kim 1,3,4* \\ ${ }^{1}$ Molecular Imaging Research Center, Korea Cancer Center Hospital, Korea Institute of Radiological and Medical \\ Sciences (KIRAMS), Seoul, Korea \\ ${ }^{2}$ Department of Nuclear Medicine, Korea Cancer Center Hospital, Korea Institute of Radiological and Medical \\ Sciences (KIRAMS), Seoul, Korea \\ ${ }^{3}$ Korea Drug Development Platform Using Radio-Isotope (KDePRI), Seoul, Korea \\ ${ }^{4}$ Radiologcial and Medico-Oncological Sciences, University of Science and Technology (UST), Seoul, Korea \\ Email: $\underline{\text { ilhan@kirams.re.kr, }}{ }^{*}$ kjs@kirams.re.kr
}

Received 21 August 2015; accepted 10 October 2015; published 13 October 2015

Copyright (C) 2015 by authors and Scientific Research Publishing Inc.

This work is licensed under the Creative Commons Attribution International License (CC BY).

http://creativecommons.org/licenses/by/4.0/

(c) (i) Open Access

\section{Abstract}

Objective: Although Iodine-125 (125I) has been widely used for in vitro studies because of its relatively long half-life (60.1 days), 125 I imaging is limited because of its low energy ( 27 - $35 \mathrm{keV}$ ), even in an animal-dedicated system. In this study, imaging characteristics of ${ }^{125}$ I were assessed using a small animal-dedicated imaging system and clinical scanner. Methods: Using the Siemens Inveon and Siemens Symbia TruePoint systems, imaging characteristics such as resolution, sensitivity, and image quality were compared. Mouse high resolution (MHR-0.5), mouse general purpose (MGP-1.0), and mouse high sensitivity (MHS-2.0) collimators were used for the Inveon scanner, and low energy high-resolution (LEHR) and low energy all-purpose (LEAP) collimators were used for the Symbia TruePoint. For animal imaging, $16.8 \mathrm{MBq}$ of ${ }^{125} \mathrm{I}$ was administered to BALB/c mice intravenously, and the planar image and single-photon emission computed tomography (SPECT) were obtained using both scanners. Results: The resolution of ${ }^{125}$ I for the Inveon scanner was 3.98 $\mathrm{mm}$ full width at half maximum (FWHM) at a $30-\mathrm{mm}$ distance with the MHR-0.5 collimator, and the value of Symbia scanner was $8.72 \mathrm{~mm}$ FWHM at a $30-\mathrm{mm}$ distance with the LEHR collimator. The sensitivity of ${ }^{125}$ I for the Inveon scanner was $21.87 \mathrm{cps} / \mathrm{MBq}$, and the value for the clinical scanner was $30.55 \mathrm{cps} / \mathrm{MBq}$. The planar images of mice were successfully obtained at the level of evaluat-

\footnotetext{
${ }^{*}$ Corresponding authors. Authors with equal contributions.
} 
ing specific binding in both scanners. Conclusion: ${ }^{125}$ I small animal imaging can be achieved with a clinical scanner. This result may enhance the utilization of ${ }^{125}$ I small animal imaging using a clinical scanner.

Keywords

\section{I-125, Animal Imaging, Performance Measurement}

\section{Introduction}

Iodine-125 ( ${ }^{125}$ I) has been widely used for in vitro research because of its relatively long half-life (60.1 days). Various ${ }^{125}$ I-labeled nucleic acids, antibodies, and ligands are commercially available or easily made in the laboratory using commercially available reagents and kits [1] [2]. Recently, in vivo ${ }^{125}$ I imaging methods were developed, and both in vivo and in vitro experiments were performed using the same ${ }^{125}$ I-labeled tracers [3]-[7]. However, in vivo imaging in animals using ${ }^{125} \mathrm{I}$ is limited because of its low energy (27 - $35 \mathrm{keV}$ ) compared to Technetium-99m $\left({ }^{99 \mathrm{~m}} \mathrm{Tc}\right)$ or Iodine-123 $\left({ }^{123} \mathrm{I}\right)$ [8].

Although the clinical scanner was designed for imaging human patients, the clinical scanner has been used for animal imaging. Small animal imaging using a clinical scanner with a pinhole collimator and ${ }^{99 \mathrm{~m}} \mathrm{Tc}$ or ${ }^{123} \mathrm{I}$ has been reported [6] [9]-[12]. Although a small animal-dedicated scanner provides high resolution (submillimeter range), the clinical scanner is ready to use in clinics. Another advantage of the clinical scanner is the large field of view.

In this study, we investigated the feasibility for ${ }^{125}$ I imaging using a clinical scanner without a pinhole collimator and compared its performance characteristics to a small animal scanner.

\section{Materials and Methods}

\subsection{System Description}

The Symbia TruePoint (Siemens Medical Solutions USA, Inc., Knoxville, TN, USA) clinical single-photon emission computed tomography (SPECT)/computed tomography (CT) imaging system designed for human patients was used to assess ${ }^{125}$ I characteristics. Low energy high-resolution (LEHR) and low energy all-purpose (LEAP) collimators were used. Each collimator consisted of 148 holes with a 1.11-mm diameter and 90 holes with a 1.45-mm diameter across the flats. Each of the two detector heads of the Symbia TruePoint SPECT system consisted of a 15.8-mm crystal thickness and a SPECT field of view of $40 \mathrm{~cm}$ (axial) by $50 \mathrm{~cm}$ (diameter).

The Siemens Inveon small animal positron emission tomography (PET)/SPECT/CT imaging system (Siemens Medical Solutions USA, Inc.) was used to assess the performance of ${ }^{125}$ I. Mouse high resolution (MHR), mouse general purpose (MGP), and mouse high sensitivity (MHS) collimators with pinhole diameters of $0.5 \mathrm{~mm}, 1.0$ $\mathrm{mm}$, and $2.0 \mathrm{~mm}$ across the flats, respectively, were used. The Inveon SPECT/CT system consisted of two detector heads, an active area of $15 \mathrm{~cm} \times 15 \mathrm{~cm}$, [NaI(Tl)] scintillator crystal material, and 4624 crystal elements.

\subsection{Spatial Resolution and Sensitivity}

For the measurement of spatial resolution and sensitivity, a 1-mm point source was made using capillary tubes. The activities of ${ }^{125} \mathrm{I}$ and ${ }^{99 \mathrm{~m}} \mathrm{Tc}$ were $2.2 \mathrm{MBq}$ and $3.3 \mathrm{MBq}$, respectively. The data acquisition time was $600 \mathrm{~s}$. To determine the spatial resolution, profiles through peaks in count distributions were drawn in two orthogonal directions in middle slices. For each profile, full width at half maximum (FWHM) was measured by linearly interpolating the adjacent pixels at half or tenth of their maxima in the profile.

\subsection{Image Quality}

For the assessment of image quality, the National Electrical Manufacturers Association (NEMA) NU 4 Image Quality (IQ) Phantom was used [13]-[15]. The NU 4 IQ phantom is a cylindrical chamber that consists of three parts: five fillable rods with diameters of $1,2,3,4$, and $5 \mathrm{~mm}$ arranged in the top part; a uniform region in the 
middle part; and two cold components with a 10-mm diameter attached to the bottom part. The top and bottom regions are filled with nonradioactive water and air, respectively. The nonuniformity, recovery coefficient, and spillover ratio (SOR) were assessed using the NU 4 IQ phantom [14]. The initial activities for ${ }^{99 \mathrm{~m}} \mathrm{Tc}$ and ${ }^{125} \mathrm{I}$ were $11.87 \mathrm{MBq}$ and $11.36 \mathrm{MBq}$, respectively.

\subsection{Data Acquisition}

The step-and-shoot mode was used for data acquisition. The acquisition time for each view was $100 \mathrm{~s}$, total imaging time was $3200 \mathrm{~s}$, rotation degree was $180^{\circ}$, degree of configuration was $180^{\circ}$, and magnification factor was 3.2 .

\subsection{Reconstruction}

The ordered subset expectation maximization three-dimensional (OSEM-3D) algorithm was used in the small animal scanner, and the Flash 3D algorithm was used in the clinical scanner. The number of iterations was 8 , and the number of sets was 4 . The windows for ${ }^{125} \mathrm{I}$ and ${ }^{99 \mathrm{~m}} \mathrm{Tc}$ were $35 \pm 11 \mathrm{keV}$ and $140 \pm 14 \mathrm{keV}$, respectively.

\subsection{Animal Studies}

Animal study was carried out in accordance with guideline provided by the Institutional Animal Care and Use Committee (IACUC) and Institutional review board (IRB) in Korea Institute of Radiological and Medical Sciences. In addition, all experimental protocols were approved by IACUC and IRB in Korea Institute of Radiological and Medical Sciences.

${ }^{125}$ I was injected into BALB/c mice (weight: 20 g, female, SLC JAPAN) and the mice were scanned for 1 hour after injection. Planar mode imaging and SPECT mode imaging were performed. The 1.0-MGP collimator was used in the small animal scanner, and the LEAP collimator was used in the clinical scanner. The activity concentrations for the animal scanner and clinical scanner were 18.9 MBq and 14.4 MBq, respectively.

In vivo radiolabeled antibody imaging was assessed using a human breast cancer mouse model. KPL-4 and MDA-MB-231 xenografts were established in female nude mice (6 weeks) began by subcutaneously implanting approximately $5 \times 10^{6} \mathrm{KPL}-4$ cells in the right hind leg and $5 \times 10^{6} \mathrm{MDA}-\mathrm{MB}-231$ cells in the left hind leg. The size of the HER2-positive KPL-4 tumors and the HER2-negative MDA-MB-231 tumors were approximately $300 \mathrm{~mm}^{3} .{ }^{125}$ I-labeled HER2-targeting antibody was injected, and its activity concentration was $8.81 \mathrm{MBq}$.

\section{Results}

\subsection{Spatial Resolution}

Figure 1(a) shows the mean resolution as a function of source-to-collimator distance. The resolution measurements using ${ }^{125}$ I point sources are summarized in Figure 1(a). At source-to-collimator distances of 25, 30, and $35 \mathrm{~mm}$, the spatial resolutions of ${ }^{125} \mathrm{I}$ measured using the Inveon scanner were 3.32, 3.98, and $4.20 \mathrm{~mm}$ full width at half maximum (FWHM), respectively, using the MHR 0.5-mm pinhole (MHR-0.5) collimator; 3.98, 4.42, and 4.86 mm FWHM, respectively, using the MGP 1.0-mm pinhole (MGP-1) collimator; and 4.35, 4.88, and $5.43 \mathrm{~mm}$ FWHM, respectively, using the MHS 2.0-mm pinhole (MHS-2.0) collimator.

At source to collimator distances of 25,30 , and $35 \mathrm{~mm}$, the resolutions of ${ }^{125}$ I measured using the Symbia TruePoint system were 8.28, 8.72, 8.89 mm FWHM, respectively, using the LEHR collimator and 9.06, 9.19, and $9.25 \mathrm{~mm}$ FWHM, respectively, using the LEAP collimator.

Figure 1(b) shows the spatial resolution of ${ }^{99 \mathrm{~m}} \mathrm{Tc}$ point sources. The results were similar to a previous study and specification sheets provided by the manufacturer [15]-[17].

\subsection{Sensitivity}

Figure 2 shows the sensitivity of ${ }^{125} \mathrm{I}$ point sources as a function of source-to-collimator distance. At sourceto-collimator distances of 25, 30, and $35 \mathrm{~mm}$, the sensitivities for the Inveon scanner using the MHR-0.5 collimator were 29.49, 21.87, and $17.12 \mathrm{cps} / \mathrm{MBq}$, respectively; the MGP-1.0 collimator were 64.41, 44.37, and $32.69 \mathrm{cps} / \mathrm{MBq}$, respectively; and the MHS-2.0 collimator were 163.42, 116.88, and $87.18 \mathrm{cps} / \mathrm{MBq}$, respectively. 


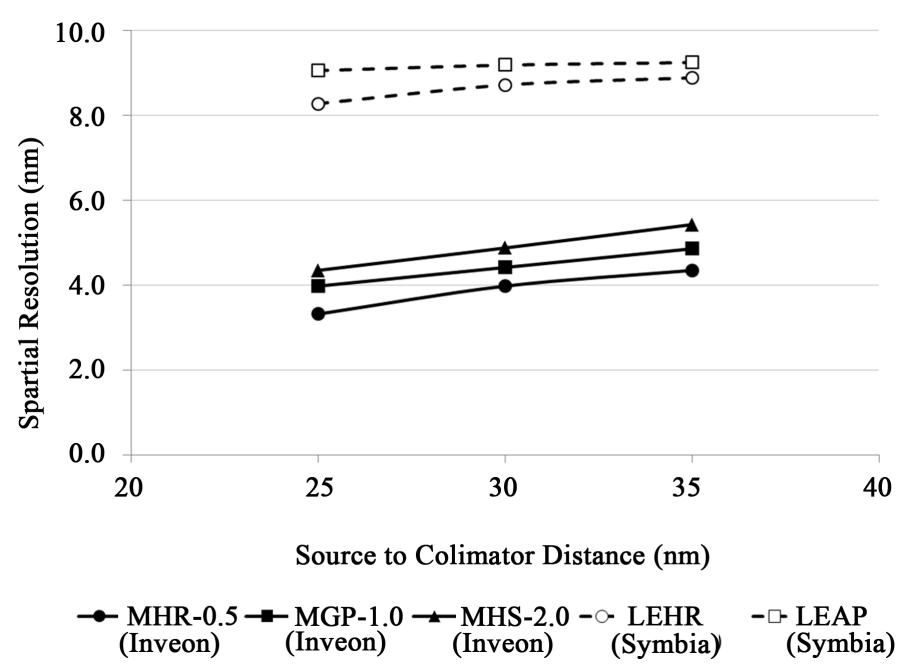

(a)

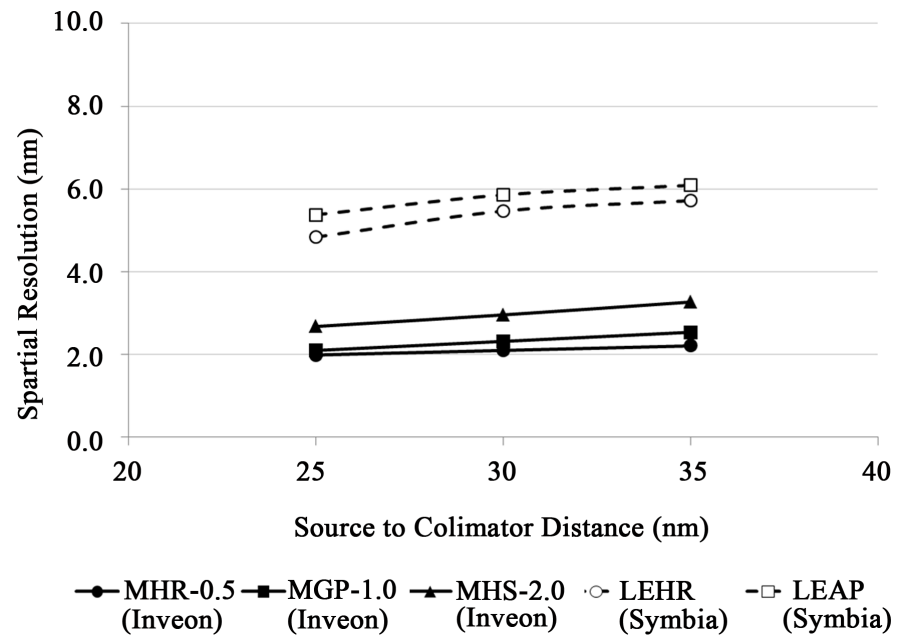

(b)

Figure 1. The resolution of the clinical scanner compared to the small animal scanner presented as a function of the source-to-collimator distance with each collimator. (a) Idoine-125 $\left({ }^{125} \mathrm{I}\right)$ point source and (b) Technetium-99m $\left({ }^{99 \mathrm{~m}} \mathrm{Tc}\right)$ point source.

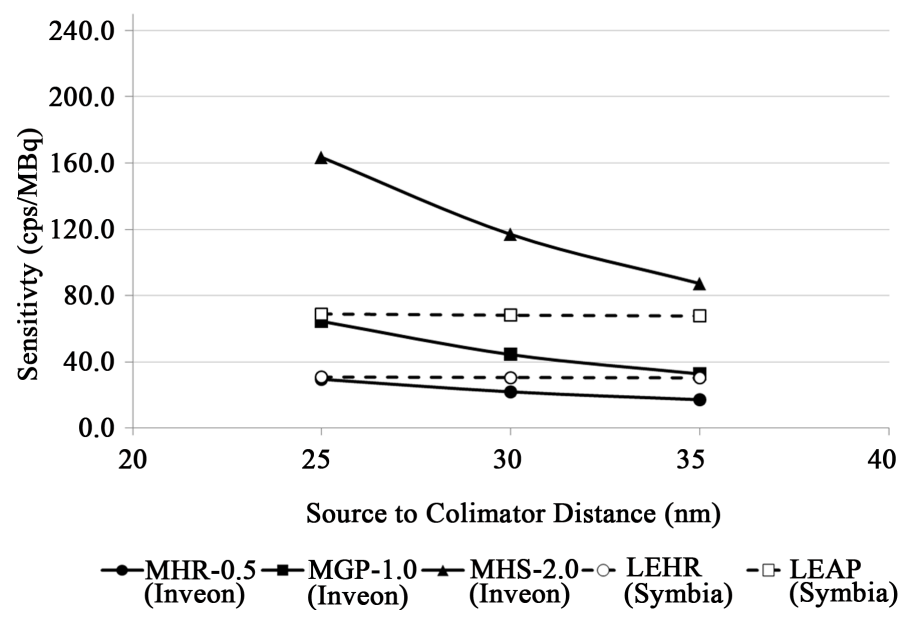

(a) 


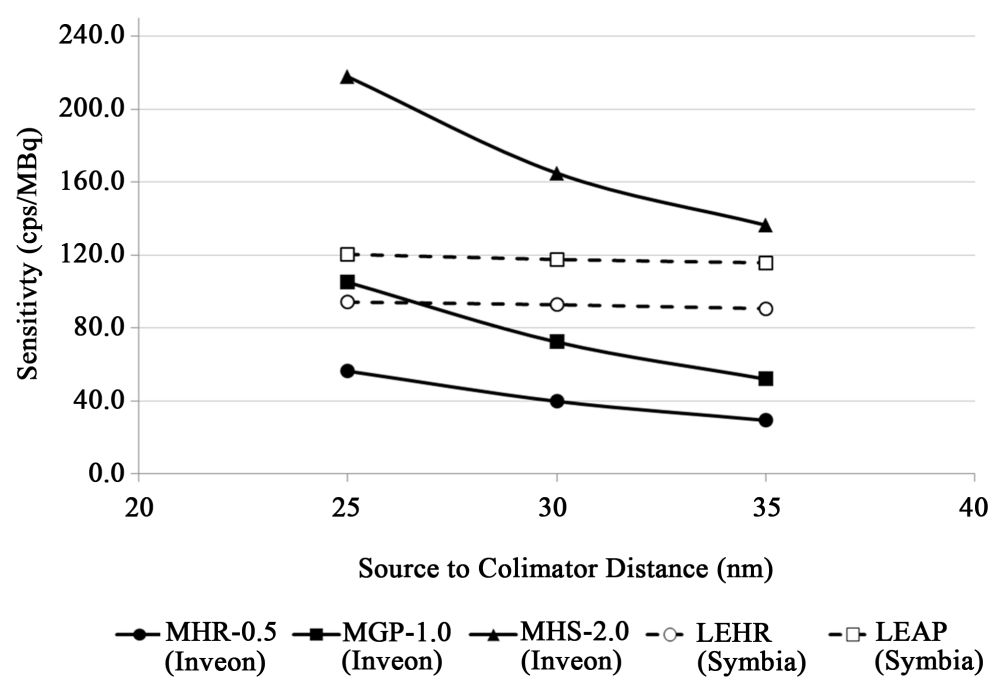

(b)

Figure 2. The sensitivity of the clinical scanner compared to the small animal scanner presented as a function of the source-to-collimator distance with each collimator. (a) Iodine-125 $\left({ }^{125} \mathrm{I}\right)$ point source and (b) Technetium-99m $\left({ }^{99 \mathrm{~m}} \mathrm{Tc}\right)$ point source.

At source-to-collimator distances of 25, 30, and $35 \mathrm{~mm}$, the sensitivities for the Symbia TruePoint were 30.83, 30.55, and $30.35 \mathrm{cps} / \mathrm{MBq}$, respectively, using the LEHR collimator and 68.89, 68.13, and $67.68 \mathrm{cps} / \mathrm{MBq}$, respectively, using the LEAP collimator.

Figure 2(b) shows the sensitivity using ${ }^{99 \mathrm{~m}} \mathrm{Tc}$ point sources. These results were similar to a previous study [15]-[17].

\subsection{Nonuniformity}

Figure 3(a) shows the nonuniformity (\%STD ${ }_{\text {unif }}$ ) of ${ }^{125}$ I measured by the NU 4 IQ phantom. The nonuniformities measured using the Inveon scanner were $87.97 \%$, 29.74\%, and $14.72 \%$ with the MHR-0.5, MGP-1.0, and MHS-2.0 collimators, respectively. The nonuniformities measured using the Symbia TruePoint scanner were $46.32 \%$ and $17.86 \%$ with the LEHR and LEAP collimators, respectively. These results indicated that uniformity enhanced by acquired counts.

The nonuniformity of ${ }^{99 \mathrm{~m}}$ Tc measured by the NU 4 IQ phantom was similar to ${ }^{125}$ I (Figure 3(b)).

\subsection{Recovery Coefficient}

Figure 4 shows the recovery coefficient of each hot rod in a cold background as a function of rod diameter. However, there are two problems, image noise and resolution of the clinical scanner, for evaluating recovery coefficients. Generally, the theoretical maximum value of $\mathrm{RC}_{\mathrm{rod}}$ is 1.0 , but it can be more than 1.0 because of image noise. The resolution of the clinical scanner was $8.89 \mathrm{~mm}$ FWHM in the LEHR collimator and $9.25 \mathrm{~mm}$ FWHM in the LEAP collimator for ${ }^{125} \mathrm{I}$ point sources. Therefore, detecting hot rods $(1.0-5.0 \mathrm{~mm})$ was impossible. For these reasons, calculation of this parameter on the clinical scanner was incomplete and was predicted by the position of the hot rod in the object based on the physical dimensions of the phantom.

\subsection{Spillover Ratio}

Figure 5(a) shows the results of SOR using the ${ }^{125}$ I-filled NU 4 IQ phantom. The SOR wat $_{\text {was }} 20.41 \%, 19.79 \%$, $16.95 \%$ in the Inveon scanner with the MHR-0.5, MGP-1.0, and MHS-2.0 collimators, respectively. The SOR air in the Inveon scanner was $14.71 \%, 12.65 \%$, 8.96\%, with the MHR-0.5, MGP-1.0, and MHS-2.0 collimators, respectively. The $\mathrm{SOR}_{\text {wat }}$ in the Symbia TruePoint scanner was $36.30 \%$ and $32.30 \%$ with the LEHR and LEAP collimators, respectively. The SOR air $_{\text {in }}$ the Symbia TruePoint scanner was $28.14 \%$ and $23 \%$ with the LEHR and LEAP collimators, respectively.

Figure 5(b) shows the SOR results measured by ${ }^{99 \mathrm{~m}}$ Tc-filled NU 4 IQ phantom. 


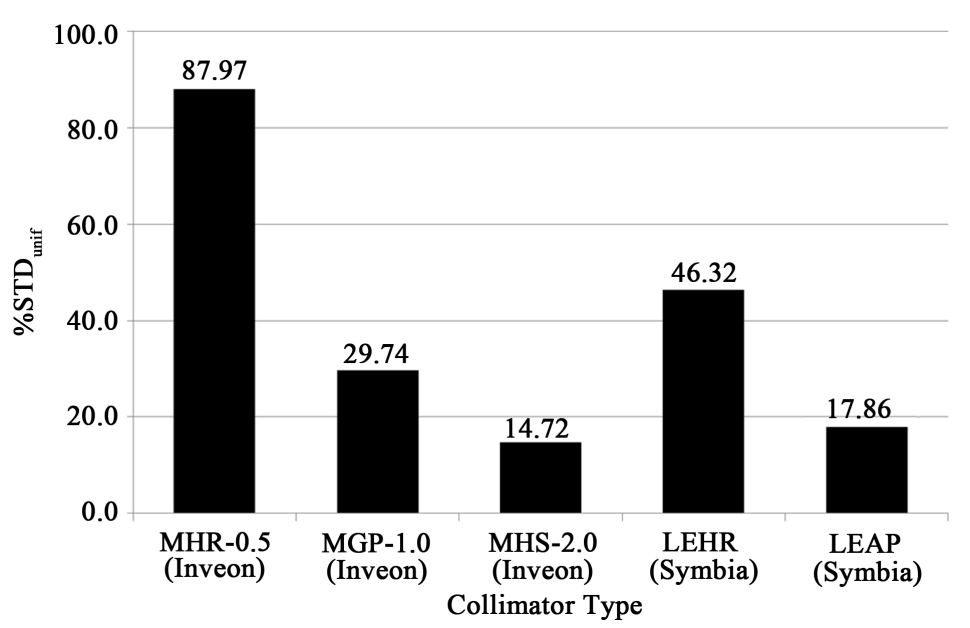

(a)

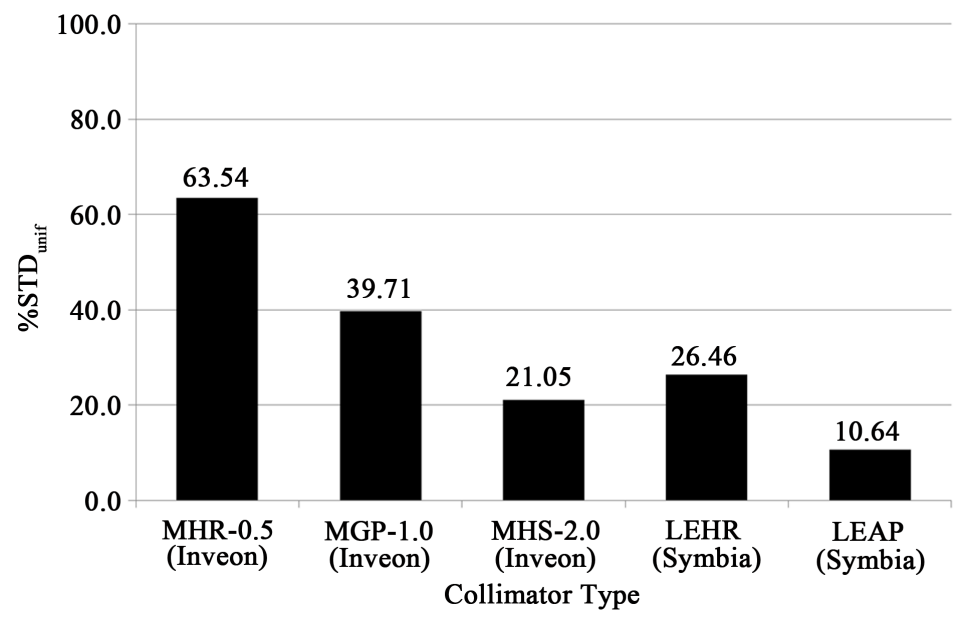

(b)

Figure 3. Nonuniformity (\%STD $\mathrm{Snif}_{\text {) }}$ ) of the clinical scanner compared to the small animal scanner. (a) NU 4 Image Quality (IQ) Phantom filled with Iodine-125 ( $\left.{ }^{125} \mathrm{I}\right)$ and (b) Technetium-99m $\left({ }^{99 \mathrm{~m}} \mathrm{Tc}\right)$.

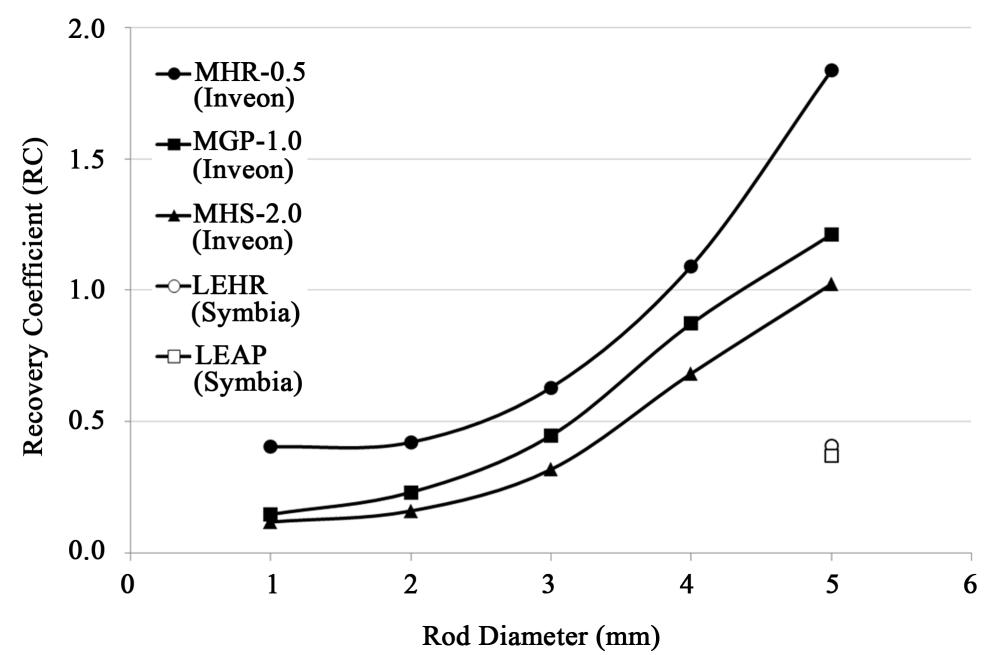

Figure 4. Recovery coefficient for Iodine-125 $\left({ }^{125}\right.$ I)-filled rods of 1, 2, 3, 4, and 5 mm diameter. 


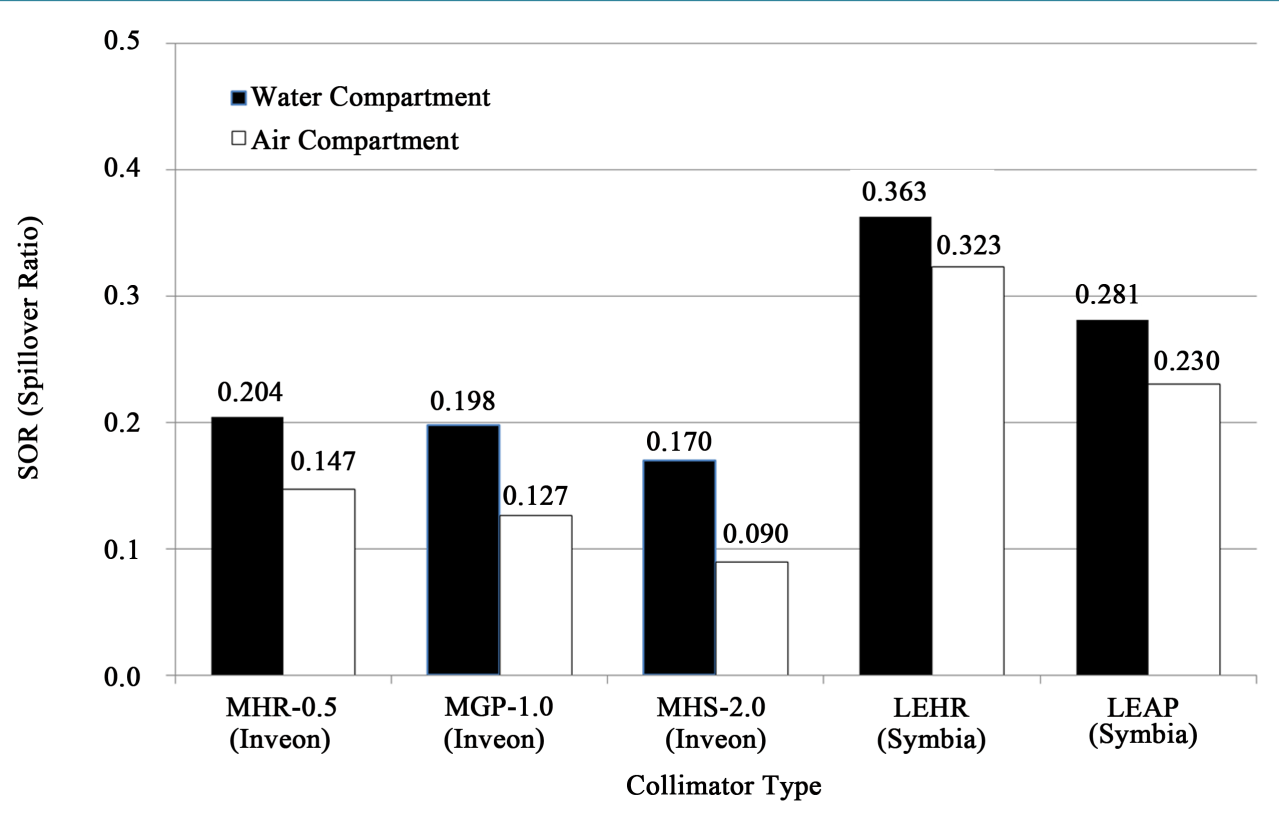

(a)

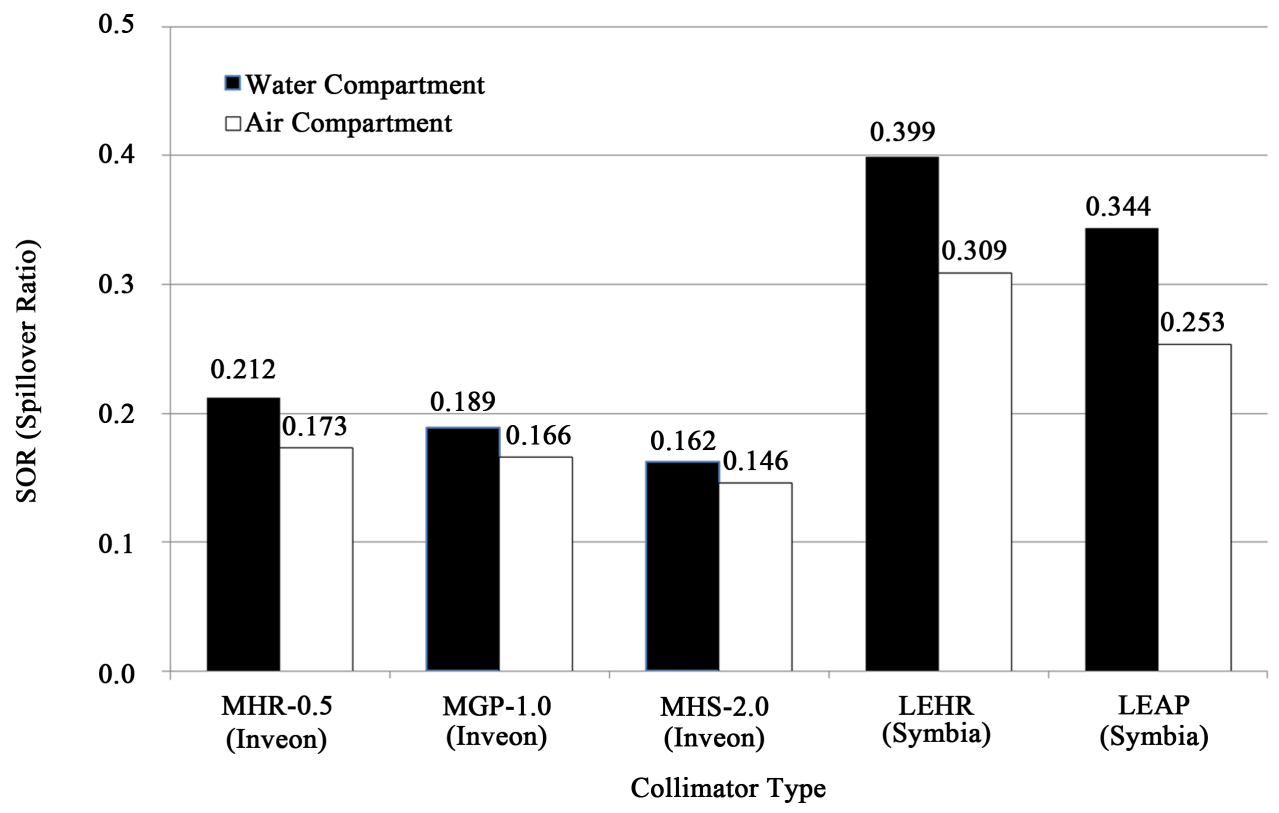

(b)

Figure 5. Spillover ratios (SORs) for cold-water and air compartments. (a) NU 4 Image Quality (IQ) Phantom filled with Iodine-125 $\left({ }^{125} \mathrm{I}\right)$ and $(\mathrm{b})$ Technetium-99m $\left({ }^{99 \mathrm{~m}} \mathrm{Tc}\right)$.

\subsection{Animal Studies}

\section{Small Animal Imaging}

Figure 6(a) and Figure 6(b) show the ${ }^{125}$ I images of a BALB/c mouse acquired by the Inveon scanner using the planar and SPECT modes, respectively. Figure 6(c) and Figure 6(d) show the ${ }^{125}$ I images of a BALB/c mouse acquired by the Symbia TruePoint scanner using the planar and SPECT modes, respectively.

Figure 6(e) shows the ${ }^{125}$ I-affibody image of human breast cancer KPL-4 and MDA-MB-231 xenograft mice using the Symbia TruePoint scanner. Two mice were scanned simultaneously using the clinical scanner at 1 hour after ${ }^{125}$ I-affibody injection. The tumors were faintly visualized as accumulation regions on the hind leg. 


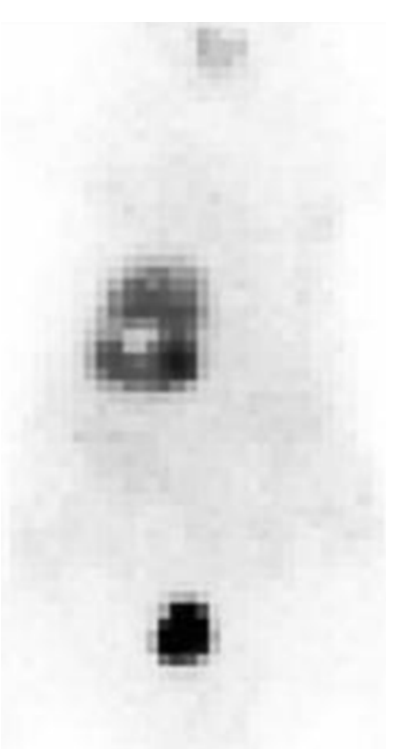

(a)

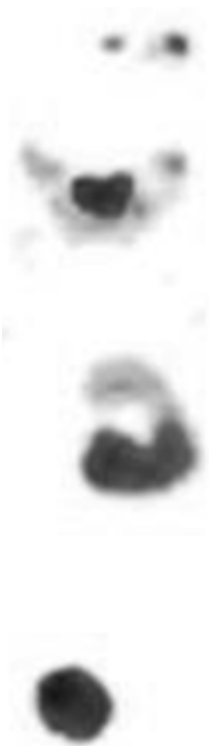

(b)

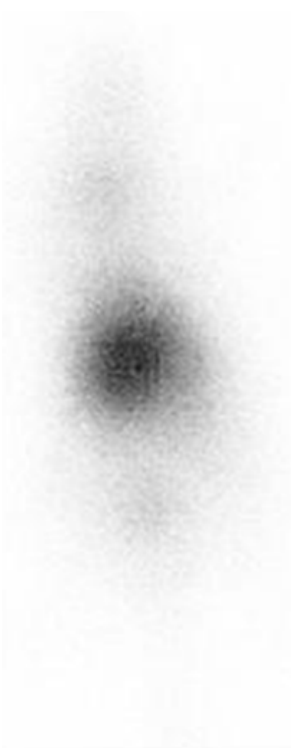

(c)

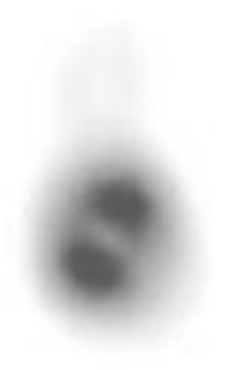

(d)

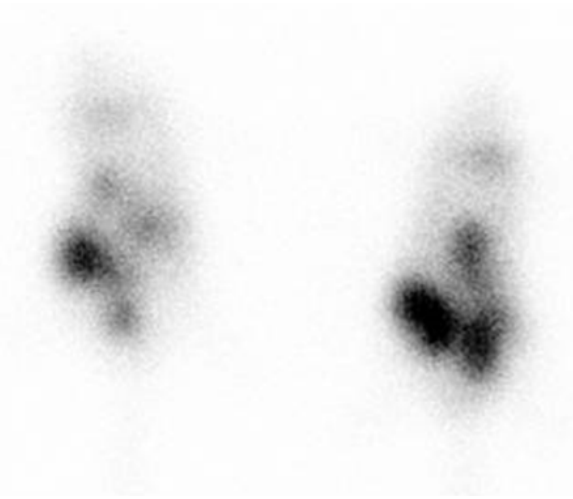

(e) Ventral

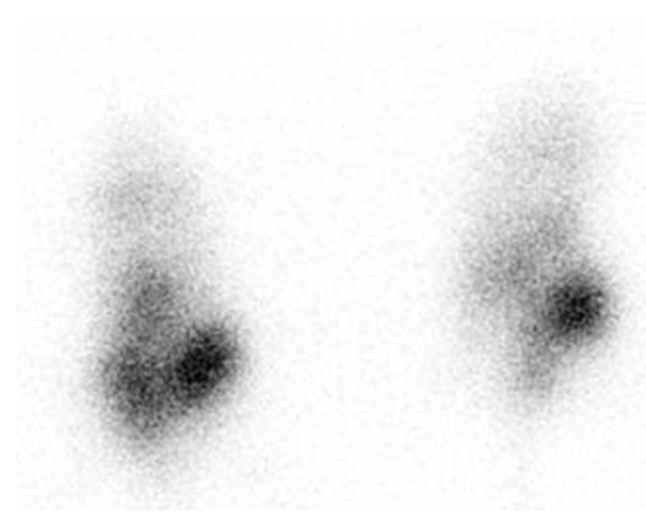

Dorsal

Figure 6. Iodine-125 $\left({ }^{125} \mathrm{I}\right)$ imaging in a BALB/c mouse. (a) Planar mode imaging with the Inveon scanner; (b) Single-photon emission computed tomography (SPECT) imaging using the Symbia TruePoint system; (c) Planar mode imaging using the Inveon scanner; (d) SPECT imaging using the Symbia TruePoint system; (e) Planar imaging using the Symbia TruePoint of human breast tumor KPL-4 and MDA-MB-231 xenograft mice after ${ }^{125}$ I labeled affibody injection.

\section{Discussion}

This comparative result of ${ }^{125}$ I showed that the sensitivity was comparable between the Inveon scanner and Symbia TruePoint scanner. An important limitation of the evaluation of the clinical scanner stemmed mostly from the source-to-collimator distance and small phantom object size. The recovery coefficient was indefinite, and the spillover ratio was overestimated in the clinical scanner. Nevertheless, the uniformity of the clinical scanner was acceptable because of the parallel collimator.

For the reasons mentioned above, attempting small animal imaging by a clinical scanner is more important than statistically analysis. Not surprisingly, the performance of the small animal scanner was undoubtedly better than that of the clinical scanner. Even small components could be distinguished with less blurring than clinical scanner. However, Figure 6(e) implies an unexpected possibility for small animal imaging beyond numeric data. Accumulation of ${ }^{125}$ I-labeled target agents in the hind legs of a xenograft mouse model of human cancer was confirmed sufficiently for the purpose in spite of planar mode imaging. These results are acquired by the daily routine methods for patient imaging in hospitals without special modifications, such as attaching pinhole collimators. 
The small animal imaging system is an important tool with great potential for development of pharmaceutical agents aimed at diagnosis and therapy. Therefore, the research demand for small animal imaging has also increased over the last decade [4]-[7]. The resolution of small animal scanners is generally in the submillimeter range. This range is a considerable improvement compared to the $8 \mathrm{~mm}$ or higher range resolution of existing clinical scanners [18] [19]. Because a high-resolution imaging system is not always available in clinical environment, clinical scanner for small animal imaging would be feasible imaging modality for animal imaging and research.

\section{Conclusion}

This study collectively showed that ${ }^{125}$ I animal imaging using the Symbia TruePoint scanner was feasible for animal imaging. The advantages of using the clinical scanner include its large field of view, which would allow imaging of 2 - 3 mice, and availability, since it could be used where a dedicated small animal scanner system is not available. Small animal imaging using a clinical scanner is more accessible and readily available. This study shows that using clinical scanner can expand small animal imaging research opportunities. Our results also showed the possibility of ${ }^{125} \mathrm{I}$ for clinical use although ${ }^{125} \mathrm{I}$ imaging was not feasible due to low energy of ${ }^{125} \mathrm{I}$ for clinical imaging.

\section{Conflict of Interest}

The authors declare that they have no conflict of interest.

\section{Supported}

This work was supported by Priority Research Centers Program through the National Research Foundation of Korea (NRF) funded by the Ministry of Education, Science and Technology (50458-2011 (PI: Ilhan Lim)). This work was supported by the Korea Science and Engineering Foundation (KOSEF) (No. 2015001667 (PI: Joo Hyun Kang) and No. 1711026888 (PI: Kook-Hyun Yu)).

\section{References}

[1] Beekman, F.J., et al. (2002) Towards in Vivo Nuclear Microscopy: Iodine-125 Imaging in Mice Using Micro-Pinholes. European Journal of Nuclear Medicine and Molecular Imaging, 29, 933-938. http://dx.doi.org/10.1007/s00259-002-0805-6

[2] Dilmanian, F.A., et al. (1990) A High Resolution SPECT System Based on a Microchannel-Plate Imagier. IEEE Transactions on Nuclear Science, 37, 687-695. http://dx.doi.org/10.1109/23.106699

[3] Kim, E.M., et al. (2009) Characterization, Biodistribution and Small-Animal SPECT of I-125-Labeled c-Met Binding Peptide in Mice Bearing c-Met Receptor Tyrosine Kinase-Positive Tumor Xenografts. Nuclear Medicine and Biology, 36, 371-378. http://dx.doi.org/10.1016/j.nucmedbio.2009.01.005

[4] Franc, B.L., et al. (2008) Small-Animal SPECT and SPECT/CT: Important Tools for Preclinical Investigation. Journal of Nuclear Medicine, 49, 1651-1663. http://dx.doi.org/10.2967/jnumed.108.055442

[5] Meikle, S.R., et al. (2005) Small Animal SPECT and Its Place in the Matrix of Molecular Imaging Technologies. Physics in Medicine and Biology, 50, R45-R61. http://dx.doi.org/10.1088/0031-9155/50/22/r01

[6] Weber, D.A., et al. (1994) Pinhole SPECT: An Approach to in Vivo High Resolution SPECT Imaging in Small Laboratory Animals. Journal of Nuclear Medicine, 35, 342-348.

[7] Herschman, H.R. (2003) Molecular Imaging: Looking at Problems, Seeing Solutions. Science, 302, 605-608. http://dx.doi.org/10.1126/science.1090585

[8] Hwang, A.B., et al. (2008) Assessment of the Sources of Error Affecting the Quantitative Accuracy of SPECT Imaging in Small Animals. Physics in Medicine and Biology, 53, 2233-2252. http://dx.doi.org/10.1088/0031-9155/53/9/002

[9] Scherfler, C. and Decristoforo, C. (2006) Small Animal Imaging Using a Conventional Gamma Camera Exemplified in Studies on the Striatal Dopaminergic System. Nuclear Medicine Review Central East Europe, 9, 6-11.

[10] Aide, N., et al. (2010) High Throughput Static and Dynamic Small Animal Imaging Using Clinical PET/CT: Potential Preclinical Applications. European Journal of Nuclear Medicine and Molecular Imaging, 37, 991-1001. http://dx.doi.org/10.1007/s00259-009-1352-1

[11] Khalil, M.M., Tremoleda, J.L., Bayomy, T.B. and Gsell, W. (2011) Molecular SPECT Imaging: An Overview. Inter- 
national Journal of Molecular Imaging, 2011, Article ID: 796025. http://dx.doi.org/10.1155/2011/796025

[12] Umeda, I.O., Tani, K., Tsuda, K., Kobayashi, M., Ogata, M., Kimura, S., et al. (2012) High Resolution SPECT Imaging for Visualization of Intratumoral Heterogeneity Using a SPECT/CT Scanner Dedicated for Small Animal Imaging. Annals of Nuclear Medicine, 26, 67-76. http://dx.doi.org/10.1007/s12149-011-0542-7

[13] Rowland, D.J. and Cherry, S.R. (2008) Small-Animal Preclinical Nuclear Medicine Instrumentation and Methodology. Seminars in Nuclear Medicine, 38, 209-222. http://dx.doi.org/10.1053/j.semnuclmed.2008.01.004

[14] Harteveld, A.A., Meeuwis, A.P.W., Disselhorst, J.A., Slump, C.H., Oyen, W.J.G., Boerman, O.C. and Visser, E.P. (2011) Using the NEMA NU 4 PET Image Quality Phantom in Multipinhole Small-Animal SPECT. Journal of Nuclear Medicine, 52, 1646-1653. http://dx.doi.org/10.2967/jnumed.110.087114

[15] Medical, S. (Ed.) (2005) Symbia ${ }^{\mathrm{TM}}$ TruePoint SPECT/CT System Specifications.

[16] Medical, S. (Ed.) (2006) Inveon ${ }^{\mathrm{TM}}$ System Specifications Data.

[17] Macey, D.J., DeNardo, G.L., DeNardo, S.J. and Hines, H.H. (1986) Comparison of Low- and Medium-Energy Collimators for SPECT Imaging with Iodine-123-Labeled Antibodies. Journal of Nuclear Medicine, 27, 1467-1474.

[18] Magota, K., Kubo, N., Kuge, Y., Nishijima, K.-I., Zhao, S.J. and Tamaki, N. (2011) Performance Characterization of the Inveon Preclinical Small-Animal PET/SPECT/CT System for Multimodality Imaging. European Journal of Nuclear Medicine and Molecular Imaging, 38, 742-752. http://dx.doi.org/10.1007/s00259-010-1683-y

[19] Kojima, A., Matsumoto, M., Takahashi, M., Hirota, Y. and Yoshida, H. (1989) Effect of Spatial Resolution on SPECT Quantification Values. Journal of Nuclear Medicine, 30, 508-514. 